\title{
Object Oriented Parallel Programming Model on a Network of Workstations
}

\author{
B. Suresh and R. Nadarajan \\ Department of Mathematics and Computer Applications, PSG College of Technology, \\ Peelamedu, Coimbatore-641 004, TamilNadu, India \\ \{suresh_psgtech, nadarajan_psg\} @yahoo.co.in
}

\begin{abstract}
Parallel computing in network of workstations is receiving lot of attentions from the research community. However, still it is faced with many practical difficulties. One of the major problems is the lack of high level programming model. Many programming languages provide an efficient system, but employ a relatively low level programming model and rather too complex to be used in network environment. The object oriented system paradigm in software engineering has wide acceptance and provides support for the construction of modular and reusable program components and it is attractive for the design of large and complex serial programs. To address the previous said problem, in this paper we present a flexible, easy to use, and an effective object oriented parallel programming model by amalgamating the object orientation paradigm and parallelism. It is developed on top of PVM. This model exhibits a very convenient and natural programming style and provides functionality similar to $\mathrm{C}++$.
\end{abstract}

Keywords. Object oriented programming, Parallel programming, Object oriented parallel programming.

\section{Introduction}

The object oriented paradigm provides support for the construction of modular and easily reusable program components and has proven to be useful for the design of large and complex system. In object oriented programming a system is described as a collection of objects. An object is an integrated unit of data and procedures, called methods acting on such data. The data in an object are stored in variables whose contents can be changed by assignment statements, present in the methods. It means that the variables in an object are not accessible from outside. This mechanism is a clear protocol for the exchange of information between the different objects of a programming application.

The parallel programming adopts a style, which facilitates the decomposition of a problem in to several independent tasks or processes [15]. The above said aspects of 
object oriented paradigm are potentially suitable for the field of parallel programming by treating an object as an independent task. An object and its private data are good abstraction of a process and process's local data. Public methods activated by sending messages are similar to message passing mechanism in parallel computing [13]. That is why, an object naturally becomes an autonomous programming unit or unit of execution in parallel programming environment.

Many systems have been developed by researchers on object oriented parallel programming by extending C++ (See Section 4: Related works) and defined many new notations to include specific constructs to express the parallelism. These new notations are burden to the programmers in perspective of learning its functionalities and these systems normally fail to maintain conventional programming style.

In this paper, a new object oriented parallel programming model OP++ has been proposed. We focus more on this model to give more casualty and natural programming style as like $\mathrm{C}++$ when compared to any existing system for parallel programming in network of workstations.

This paper is organized as follows: In Section 2, description about our programming model is given. In Section 3, the working principle of our model is discussed. In Section 4, some existing systems that are closely related to our model have been analyzed and in Section 5, an example application program for our model is presented.

\section{Programming Model}

$\mathrm{OP}++$ is an object oriented parallel programming language based on $\mathrm{C}++[17]$. The basic idea of $\mathrm{OP}++$ is to view an object as an independent or individual process and its member functions are decomposed computational part of the problem to provide an efficient parallel execution.

\subsection{The Process Class}

The responsibilities assigned to the programmers are to write $\mathrm{C}++$ class(es), which are decomposed part(s) to warrant parallel execution. This is accomplished by deriving the written $\mathrm{C}++$ class(es) from the built-in class called "Process". This mechanism provides "is-a" relationship between the class Process (base class) and the user written class (derived class). The class Process is a general class whose behavior is inherited by the derived class. The instance of such a class is called "process object". The process object will become an autonomous execution unit in parallel environment. The process object can be used like any other $\mathrm{C}++$ class instance.

When an object is instantiated to the class derived from the class Process, a new process is created and placed at any processing node, which has been added to the PVM [7, 5] network to implement the object's autonomous activities. This scenario will be held when the execution point reaches the constructor of the class Process at the time of process object creation. The spawned process's ID is maintained as a member data in the class Process for further communication to the corresponding 
process while any member function is invoked at the derived class of the class Process. The spawned process consists of actual definition of the class(es) that is derived from the class Process. This is discussed in detail in Section 3. So, for every process object there exists a worker process in the network. Since there is no public methods in class Process, deriving the class from it may be public, private or protected.

\subsection{Asynchronous Method Calling}

Parallelism is achieved by calling the member functions of process objects in an asynchronous manner. The statement that invoked a member function can continue its execution in parallel with the invoked member function. (i.e. the caller does not wait for the method completion and continues its execution to the consecutive statements). When the result is needed from the invoked member function, the execution has to wait until the required results are available.

The member function can call asynchronously by calling it in par \{\} block. par \{\} is a key word that we extended from compositional C++ (CC++) [2]. In our system par \{\} block implies that the method invocation are executed in parallel.

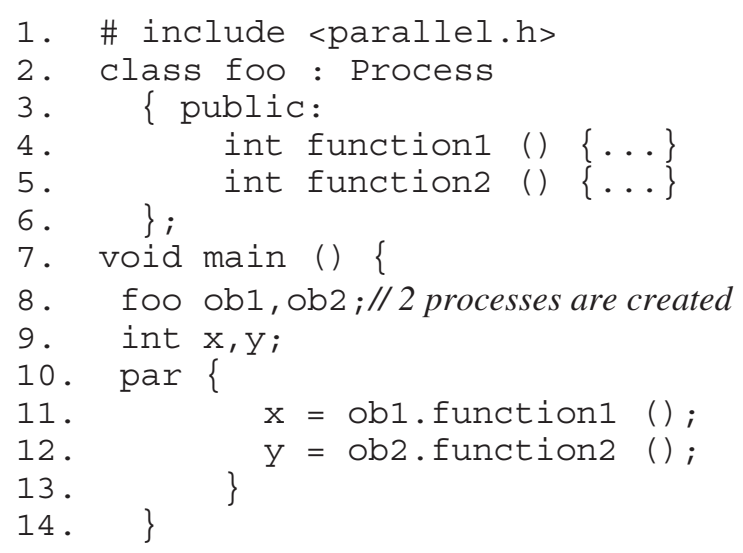

Fig 1.a. Example program

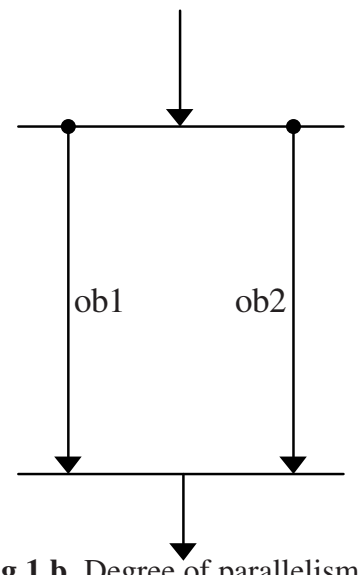

Fig 1.b. Degree of parallelism in par \{ \} block

The given program (Fig 1.a) works as follows: when object $o b 1$ and $o b 2$ are instantiated, two processes will be created on processing node in network. When the line 11 calls the member function function 1( ) that belongs to the process object $o b l$, it never waits for the return value. It continues the execution and calls another member function of another process object $o b 2$ in line 12. The value of these two member functions is received at the end of the par \{\} block. The receiving point or synchronous point is found out depending upon the data dependency that exists in the given code inside the par \{\} block. This is discussed in detail in Section 2.3 with an example. 
The member function belongs to different process object can be invoked and executed on different nodes in parallel. As shown in Fig 1.b, ob1.function1 ( ) and ob2.function 2 ( ) are executed in parallel. The only thing is no more than one member function of the given process objects will be executed at the same time. When more than one member function is called on same process object in par \{\} block it is executed in a sequential manner. The statements that are not available in par \{\} block will be executed sequentially.

\subsection{Synchronization Point}

Since the method in side the par \{\} block are invoked asynchronously, synchronization between the statements is required. The synchronization mechanism defined in our model can be described as follows. Methods invoked prior to a synchronization point can be executed in parallel with the calling methods, but only after the invoked methods have returned can the statements proceed from synchronization point.

Consider the process objects $o b 1, o b 2$ and $o b 3$. (i.e., the instance of the class derived from the class Process). The dependency between the statements in par \{\} block is observed at compile time and it produces suitable code to maximize the degree of parallelism. It is assumed that the methods used in the given example return some result, so that the dependency can be explained in more details.

$$
\begin{aligned}
& \text { par }\{ \\
& \text { 1. } \mathrm{x}=\text { obl.fun }(10,10) \text {; } \\
& \text { 2. } a=b+c \text {; } \\
& \text { 3. } y=0 b 2 \text {. fun }(10, a) \text {; } \\
& \text { 4. } z=0 \text { b3.fun }(15,10) \text {; } \\
& \text { 5. } w=\text { obl.fun }(1,2) \text {; } \\
& \text { 6. } \mathrm{C}=\mathrm{y}+\mathrm{z} \text {; } \\
& \text { \} }
\end{aligned}
$$

Fig 2.a Example code

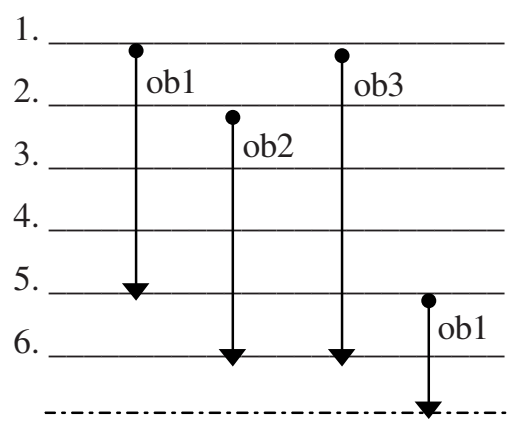

End of par \{\} block

Fig 2.b Degree of parallelism

In Fig 2.a, the methods in line 1 and line 4 are invoked because both methods were called on different process objects and there doesn't exist any dependency between these statements. The method in line 3 invoked after the execution of line 2 because the method uses the variable "a" that was defined by line 2 .

The value is received from the method of $o b l$ before the line 5 since no more than one member function will run in parallel. Now the line 5 is executed. The value is received from the method of $o b 2$ and $o b 3$ before the line 6 and the value is received from $o b 1$ at end of the par \{\} block (Fig 2.b). The statements that are not associated with method invocation will not run in parallel with each other (i.e line $2 \&$ line 6). And also if there is any statement like, 


$$
x=\text { ob3.fun1 (ob1.fun }(10,10) \text {, ob2.fun }(15,25))
$$

in par \{\} block, the method of different process objects $o b 1$ and $o b 2$ in object $o b 3$ are invoked and executed in parallel. After the execution of the method of $o b l$ and $o b 2$ the result is forwarded to the method of $o b 3$.

\subsection{Lifetime of a Process in a Network}

The lifetime of a process that spawned at the time of creation of a process object is based on lifetime of the process object. When an object is created the process is created and placed in the processing node of the network. When the scope of the process object goes out and its destructor is reached, it kills the corresponding process from the network. The users need not to bother about maintaining the running processes.

\subsection{Nested Parallelism}

The fundamental construct of our model is based on master/worker process model. The process that runs main () function is said to be a master process. Other processes spawned at the time of process object creation are said to be worker processes. The worker process, which itself is capable of creating some other worker process(es) and so on. This kind of parallel execution is referred as a nested parallelism [11]. The nested parallelism is accomplished by containment [17]. The containment to achieve the nested parallelism is the inclusion of instance of a class derived from the class Process as a member data in its private area of another class derived from the class Process. The given example shows the nested parallelism by means of containment.
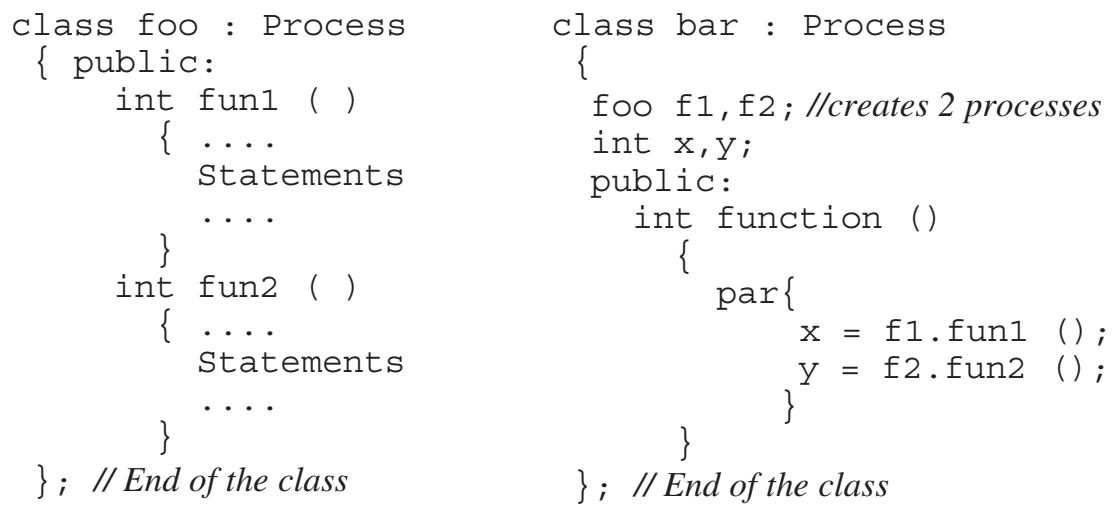

The bar class consists of two process objects that are instance of foo class. The method in a bar class calls two methods of process objects $\mathrm{f} 1 \& \mathrm{f} 2$ in par \{\} block to run it in parallel. When an object (instance of bar class) invokes this method in some other par block, this gives nested parallelism. 

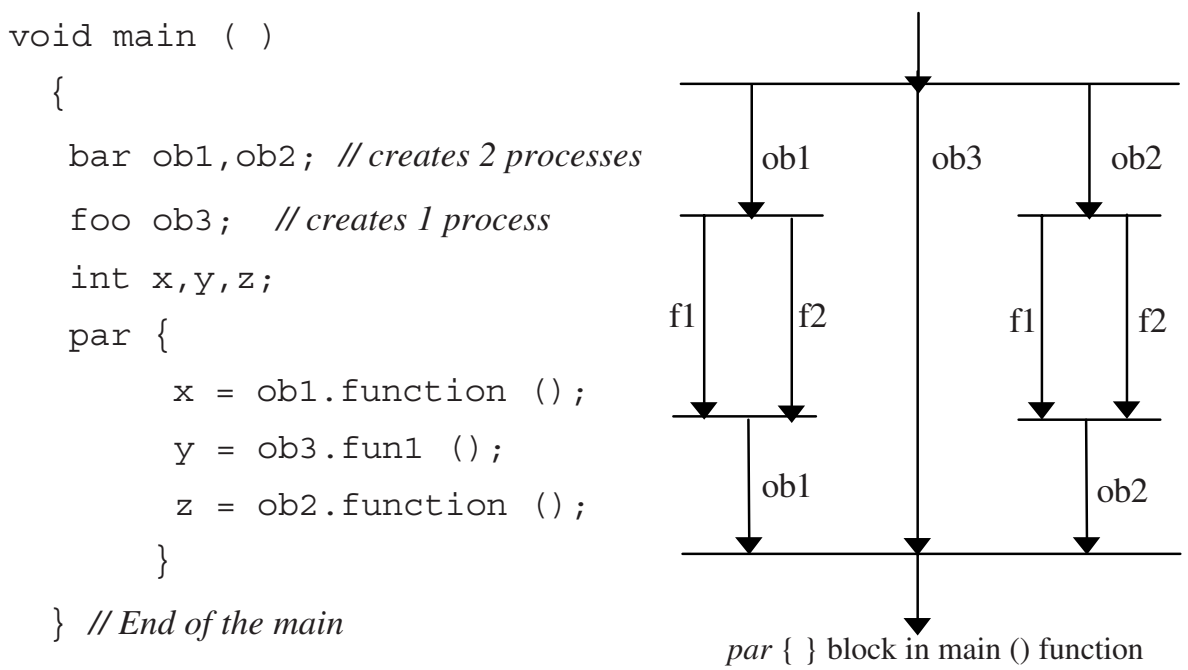

\subsection{Parallel Arithmetic Expression}

Many scientific problems contain many arithmetic expressions, which take much time to evaluate. Normally most of the arithmetic expressions may have some sub expression which doesn't affect the result of the given expression if it is evaluated in parallel. That is the result of some sub expression is not required at time of some other expression evaluation. Such type of sub expressions can be observed and safely executed in parallel. Our system provides this facility to evaluate the expression in parallel, when the operands of the expression is a process object and the operator followed by that process object in the expression are overloaded as a public method.

To illustrate the parallel expression consider the process objects $a, b, c$ and $d$. The operators succeeded to it are overloaded operators and the operands followed by the operator are parameters to the operator function.

$$
\operatorname{par}\{\mathrm{x}=((a+2) *(c / \mathrm{f}))+(d * 10)+(b / \mathrm{f})\}
$$

Step $1:(a+2),(c / f),(d * 10),(b+f)$ are computed simultaneously.

Step 2 : Result of $(a+2) *$ Result of $(c / f)$ is computed

Step 3 : Result of step $2+$ Result of $(d * 10)$ is computed

Step 4 : Result of step $3+$ Result of $(b / f)$ is computed

In the above expression when $(a+2)$ is evaluated the result of other sub expression like $(c / \mathrm{f}),(d * 10)$ and $(b / \mathrm{f})$ are not required. So these sub expressions are evaluated in parallel. That is the methods $+, /,{ }^{*}, /$ of the process objects $a, b, c$ and $d$ respectively are invoked asynchronously.

The synchronization points of the invoked methods (operator function) in an arithmetic expression strictly depend on the precedence of the operator at the 
evaluation. When the above expression is evaluated in serial, it requires seven steps, but in parallel, it is evaluated in four steps. But this gives good computational performance when large amount of computation (coarse grained) is performed in operator overloaded method.

\subsection{Inheritance and Virtual Functions}

The concepts of inheritance of object orientation raised the reusability and extendibility of existing code over other programming styles. Basically our system is based on single inheritance to provide parallel processing by derived a class from the class Process. The inheritance can continue to a class from the class derived by the class Process. OP++ also supports virtual function to give the polymorphism to the public method in conjunction with dynamic binding.

Our system supports both multi level inheritance and multiple inheritance. While using multiple inheritance, the only rule to follow is the parents of the class that emerge in multiple inheritance must derived virtually from the class Process. The reason is when any object is created to the class derived from the class Process, a unique process ID is created and maintained in a private data of the class Process. To avoid the ambiguity of the process ID in class Process, when any class is derived from more than a class that is derived from the class Process, we need to maintain a single copy of a class Process by means of virtual base class.

\section{Principle of $\mathrm{OP}++$}

A program written in $\mathrm{OP}++$ is passed through the $\mathrm{C}++$ preprocessor to extract the definition about the classes that are derived from the class Process. From the extracted information, the system creates a file whose name is filename_wrk.cpp by removing the key word par \{\} and adding appropriate calls that are related to PVM library functions. The worker file contains the actual code of the written class, which is derived from the class Process. The native $\mathrm{C}++$ compiler compiles this file to generate an executable file (worker program) and it is spawned in the processing nodes that added to the PVM network at the time of object creation. The file filename.cpp is regenerated by including necessary library functions that provide an asynchronous message passing between the worker programs and compiled to produce executable file (master program). When process object is created, it spawns the worker process and dispatches the message to it.

The message is packed of class index, function index and list of argument values. The spawned process, receipt and unpack the message and performs the desired operation, which is either the creation of a new object or a method invocation based on the value of class index and function index. After the execution of the method in the worker process the result is stored in the buffer. The master program collects the result when it needs. The indices, which are generated by this system, are unique values of every class and its public method(s) that are derived from the class Process. The node that runs the master program is said to be master node and other is worker node. 


\section{Related Works}

Investigations in amalgamating the concept of object orientation and parallel programming have already been made for several years. The classification of merging these concepts is described in [14] i.e., Parallelism in object orientation and Object orientation in parallelism. Based on these several languages are compared with respect to what objects stand for. Object may consider as process, shared passive abstract data type or encapsulation of multiple process and data.

In this section, we highlight the contribution made already in connection with our research work. We stress the criteria of object orientation in parallelism, casual programming style, flexibility and modularity. As we are interested in message passing technique, we have not considered the systems such as $\mathrm{pC}++$ [6], $\mathrm{CC}++$ [2], Distributed Processing in C++ (DPC++) [16] and DoPVM [10] which are based on distributed shared memory (DSM), which employs some complex concepts like mutex, semaphores, deadlock and critical sections. Even though the message passing technique does not involve such complex concepts, it raises the programming complexity to manage the synchronization points. So we focused on to provide an object oriented programming model based on message passing technique.

Many object oriented parallel programming systems have been developed based on DSM. But very few systems were developed using message passing. Such systems can be categorized in two ways. The first one is the system which provides object oriented library functions that supports message passing like PVM++[3], Para++[4], CPPvm[8]. These systems do not support object orientation in perspective of programming methodology and these systems are good at system and not at application level. The second one is providing object orientation in programming methodology like Dome [1] and Mentat [9, 12] that are closely relevant to our work.

Dome is based on $\mathrm{C}++$, and supports only data parallelism. It is related to our work as it is run on top of the PVM. Dome supports only SPMD (single program, multiple data) programming model. It provides a library of classes to express parallelism. When an object is instantiated to the tailor made classes the data inside the object is partitioned among the processing node of the PVM network that performs the same code on different data. There are several differences between our approach and that of Dome. Our system supports both SPMD and MPMD (multiple programs, multiple data) programming model often with in the same program. The Dome is fully constructed of tailor made classes. And also, the problem having functional parallelism cannot be implemented in Dome.

Mentat is a noteworthy portable $\mathrm{C}++$ based language. The philosophy followed in our programming model and Mentat looks related to each other. But, the principle and the execution strategy are totally different from one another. One of the major concepts is the mechanism of constructing dependency graph. In our system the dependency is observed based on process object and not depends upon the method invocation or the value of the parameters of the method of the process object. But in Mentat the dependency graph is constructed based on the value passed to the method and not by Mentat object. However, the object creation and method invocation, which makes the cost of a call unclear to the programmer. That is, if Mentat object is 
instantiated, it is not the instance itself. The new object has been created for every method invocation on that object. If $\mathrm{N}$ times the method is invoked in Mentat object it will create $\mathrm{N}$ instance of that class which may execute in parallel.

In Mentat, definition of each Mentat class must be written in a separate file and each file must be compiled separately. Even the Mentat support inheritance, the compilation order is restricted. The base class must be compiled before the derived class. This gives burden to the programmer to maintain the inheritance hierarchies at the time of compilation. But in our system, programmer can write the program in a single file that consists of any number of classes derived from the class Process. In Mentat, many new notations are introduced for the data types that cast down the causality of the programming style. One of the highlight of our system is, it allows to overload the arithmetic operators like $+,-, ।, *$ for parallel arithmetic expressions, function overloading, constructor overloading and virtual functions. Our system doesn't support friend functions, which access the private data of the object.

\section{Example Program in OP++}

In the given example code, the class MathProcess consists of two member functions that sum up and find the maximum value of the given integer array and return the results and the class is derived from the class Process. There are two arrays of process objects $o b 1$ and $o b 2$ are instantiated. The idea behind this is to split the problem array into $5 \mathrm{sub}$ arrays and it is passed to each process.

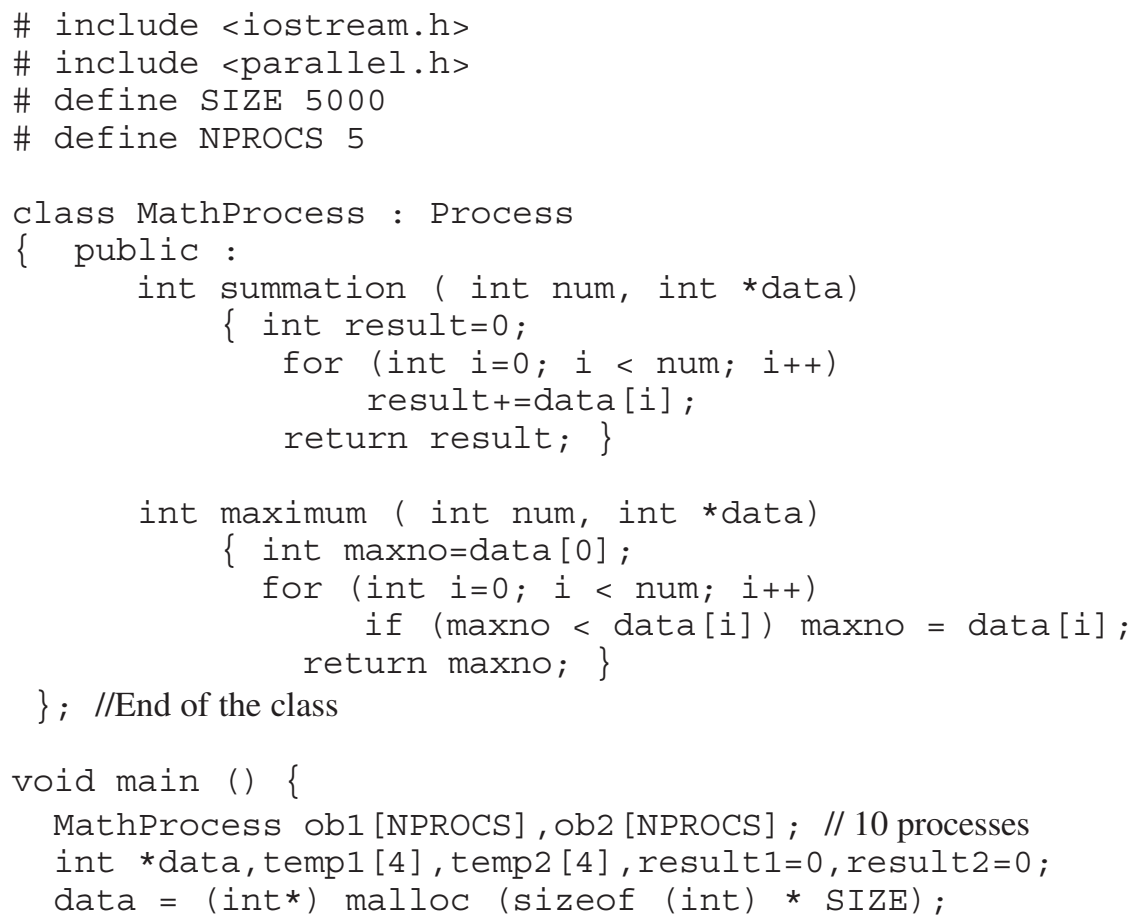




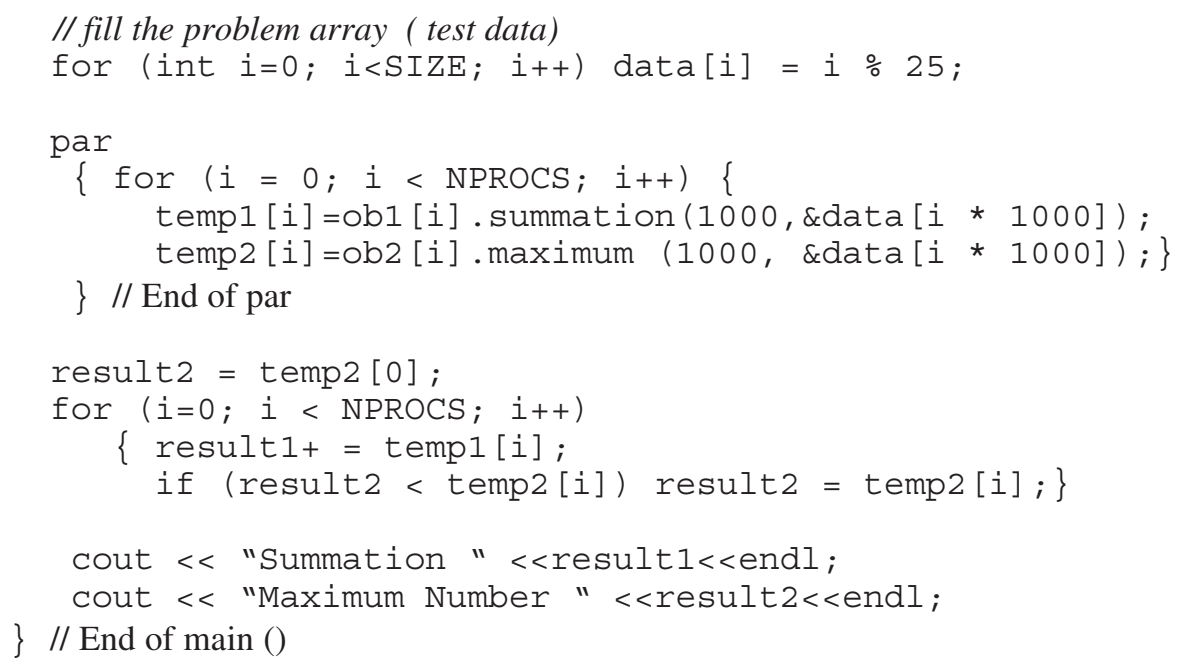

The process objects in the array $o b 1$ performs addition operation and the array $o b 2$ performs finding maximum number in an array passed to it. In par \{\} the method of process objects are invoked asynchronously by passing the array as a parameter. In method calling, the first parameter represents the number of items from the address specified followed by it or in other words the value before the address parameter specify the number of items from the given address. The array is passed by value. The copy of specified items is moved to the process. The worker process performed the operation and the result is received at the end of the par \{\} block and the intermediate result is calculated and displayed. The worker process is killed from the network when the destructor of the process object is reached.

\section{Conclusion}

In this article, we have described the object oriented parallel programming in $\mathrm{OP}++$ based on $\mathrm{C}++$ that integrates the concepts of object orientation and parallel programming. We have shown the program written in $\mathrm{OP}++$ that gives a casual programming style. The major problematic point in object oriented parallel programming is providing inheritance. Often many systems compromised in most important area like single and multiple inheritance, operator overloading and constructor overloading. But we designed our system to use all the major concepts of object oriented programming in parallel programming and also we concentrate more on minimizing the insertion of new notation that look odd. We have implemented $\mathrm{OP}++$ on network of workstations that runs on Microsoft's Windows 95/98/NT and UNIX and programmed varieties of application programs. Our experience in writing programs in $\mathrm{OP}++$ shows the benefits of object orientation that provides flexibility, modularity and casual programming style. 


\section{Acknowledgement.}

We wish to thank V. Giraga Durai for his support during this research to led this paper.

\section{Reference}

1. Adam Begulian, Erik Seligam, Michael strankey. DOME : Distributed Object Migration Environment. School of Computer Science, Carnegie Mellon University. Technical Report-CMU-CS-94-153.

2. K. Mani Chandy, Carl Kesselman. CC++:A Declarative Concurrent Object Oriented Programming Notation. A Technical Report. California Institute of Technology. March 12, 1993.

3. Daniel Cohen Laroque. $P V M++$. Institute of Computer Design \& Fault Tolerance. University of Karlsrue, September 30, 1994.

4. Oliver Coulaud, Eric Dillon. Para++ : C++ Bindings for Message Passing Libraries User Guide.March 1997. http:// www.loria.fr/para++.

5. Markus Fischer, Jack Dangarra. Experience with Windows 95/NT as a Cluster Computing Platform for Parallel Computing. Journal of Parallel and Distributed Computing Practice. 1999, Vol 2Nr2.

6. Dennis Gannon, Shelby X. Yang, Peter Beckman. User Guide for a Portable Parallel $\mathrm{C}++$. Programming System, $\mathrm{pC}++$. Deptartment of Computer Science and CICA, Indiana University, Bloominton, Indiana, USA, September 9, 1994.

7. G.A Geist, Adam Beguelin, Jack Dongarra, Weicheng Jiang, Robert Manchek, Vaidy Sunderam.PVM : Parallel Virtual Machine. A User Guide and Tutorial for Networked Parallel Computing. MIT Press, 1994.

8. Steffen Gorzig, CPPVM : C++ interface to PVM.

University of Stuttgart, Institute of Parallel and Distributed High Performance Systems.

9. A.S. Grimshaw. Introduction to Parallel Object Oriented Programming with Mentat. Department of Computer Science. University of Virginia. Report No TR-91-07, April 4, 1991.

10. C.L. Hartley and V.S. Sunderam. Programming with Shared Objects in Networked Environment, International Parallel Processing Symposium, April 1993.

11. Shih-Chen Huang. Supporting Flexible Programming Model on a Network of Workstations. PhD dissertation. New York University. January 2000.

12. Mentat 3.0 User Manual. The Mentat research group. Department of Computer Science, University of Virginia. http://www.cs.virginia.edu/ mentat

13. Janusz Niemiec. $C C++, p C++$, Charm ++ and Orca: Languages for Parallel Programming. CIS Department, Syracuse University, December 22, 1993.

14. A.A. Radenski. Object Oriented Programming \& Parallelism. Information Science Vol. 93, Issue 1-2, North Holland. Department of Computer Science. Winston Salem University. 1996.

15. Seyed H Roosta. Parallel Processing and Parallel Algorithms. Springer Publications. 1999.

16. Adre Silvetia, Rafael Avila, Marcos Barreto, Philippe Navausx. DPC++: Object Oriented Programming Applied to Cluster Computing. Institute of Informatics, Federal University of Rio Grande do Sul, Porto Algere, Brazil. First International Workshop of Project SNOW, November 23, 2001.

17. B. Stroustrup. The C++ Programming Language, Addison-Wesley, 1991. 